\title{
Recent results from CMD-3
}

F. V. Ignatov ${ }^{1,2 *}, \quad$ R. R. Akhmetshin ${ }^{1,2}, \quad$ A. N. Amirkhanov ${ }^{1,2}, \quad$ A. V. Anisenkov ${ }^{1,2}$, V. M. Aulchenko ${ }^{1,2}$, V. Sh. Banzarov ${ }^{1}$, N. S. Bashtovoy ${ }^{1}$, D. E. Berkaev ${ }^{1,2}$, A. E. Bondar ${ }^{1,2}$, A. V. Bragin ${ }^{1}$, S. I. Eidelman ${ }^{1,2,5}$, D. A. Epifanov ${ }^{1,2}$, L.B.Epshteyn ${ }^{1,2,3}$, A. L.Erofeev ${ }^{1,2}$, G. V. Fedotovich ${ }^{1,2}, \quad$ S. E. Gayazov ${ }^{1,2}, \quad$ A. A. Grebenuk ${ }^{1,2}, \quad$ S. S. Gribanov ${ }^{1,2}$, D. N. Grigoriev ${ }^{1,2,3}$, V. L. Ivanov ${ }^{1,2}, \quad$ S. V. Karpov ${ }^{1}, \quad$ V. F. Kazanin ${ }^{1,2}$, I. A. Koop ${ }^{1,2}$, A. N. Kirpotin ${ }^{1}$, A. A. Korobov ${ }^{1,2}$, A. N. Kozyrev ${ }^{1,3}$, E. A. Kozyrev ${ }^{1,2}$, P. P. Krokovny ${ }^{1,2}$, A. E. Kuzmenko ${ }^{1,2}$, A. S. Kuzmin ${ }^{1,2}$, I. B. Logashenko ${ }^{1,2}$, P. A. Lukin ${ }^{1,2}$, K. Yu. Mikhailov ${ }^{1}$, V.S. Okhapkin ${ }^{1}$, Yu. N. Pestov ${ }^{1}$, A.S. Popov ${ }^{1,2}$, G. P. Razuvaev ${ }^{1,2}$, Yu. A. Rogovsky ${ }^{1}$, A. A. Ruban ${ }^{1}$, N. M. Ryskulov ${ }^{1}$, A. E. Ryzhenenkov ${ }^{1,2}$, A. V. Semenov ${ }^{1,2}$, Yu. M. Shatunov ${ }^{1}$, P. Yu. Shatunov ${ }^{1}$, V.E. Shebalin ${ }^{1,2}$, D. N. Shemyakin ${ }^{1,2}$, B. A. Shwartz ${ }^{1,2}$, D. B. Shwartz ${ }^{1,2}$,

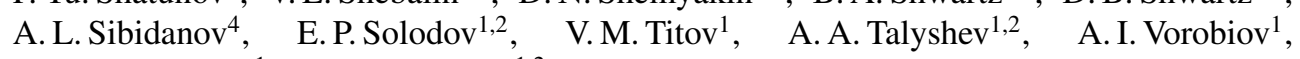
I. M. Zemlyansky ${ }^{1}$, and Yu. V. Yudin ${ }^{1,2}$

${ }^{1}$ Budker Institute of Nuclear Physics, SB RAS, Novosibirsk, 630090, Russia

${ }^{2}$ Novosibirsk State University, Novosibirsk, 630090, Russia

${ }^{3}$ Novosibirsk State Technical University, Novosibirsk, 630092, Russia

${ }^{4}$ University of Victoria, Victoria, BC, Canada V8W 3P6

${ }^{5}$ Lebedev Physical Institute RAS, Moscow, 119333, Russia

\begin{abstract}
Regular data taking with the CMD-3 at the electron-positron collider VEPP-2000 is under way since 2010. The collected data sample corresponds to about 200 inverse picobarns of integrated luminosity per detector in the energy range from 0.32 up to $2 \mathrm{GeV}$, with a goal to collect about $1 \mathrm{fb}^{-1}$ during next five years. Some of the recent results from the CMD-3 detector are discussed.
\end{abstract}

\section{Introduction}

The electron-positron collider VEPP-2000 [1] has been operating at Budker Institute of Nuclear Physics since 2010. The collider is designed to provide luminosity up to $10^{32} \mathrm{~cm}^{-2} \mathrm{~s}^{-1}$ at the maximum center-of-mass energy $\sqrt{s}=2 \mathrm{GeV}$. At present two detectors, CMD-3 [2, 3] and SND [4], are installed in the interaction regions of the collider. In 2010 both experiments started data taking. The current collected integrated luminosity is about $200 \mathrm{pb}^{-1}$ per detector. The physics program [5] includes high-precision measurements of the $e^{+} e^{-} \rightarrow$ hadrons cross sections in the wide energy range up to $2 \mathrm{GeV}$, with rich intermediate dynamics involved, studies of known and searches for new vector mesons, studies of $n \bar{n}$ and $p \bar{p}$ production cross sections near threshold and searches for exotic hadrons. It requires a detector with high efficiency for multiparticle events and good energy and angular resolutions for charged particles as well as for photons.

CMD-3 (Cryogenic Magnetic Detector) is a general-purpose detector, see Fig. 1. Coordinates, angles and momenta of charged particles are measured by the cylindrical drift chamber

\footnotetext{
*e-mail: ignatov@inp.nsk.su
} 


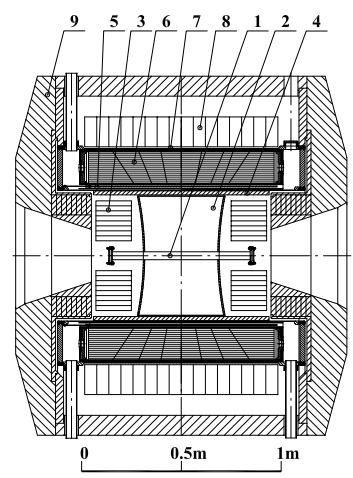

Figure 1. CMD-3 detector: 1 - beam pipe, 2 - drift chamber, 3 - BGO calorimeter, 4 Z-chamber, 5 - SC solenoid $\left(0.13 X_{0}, 13 \mathrm{kGs}\right)$, 6 - LXe calorimeter, 7 - TOF system, 8 CsI electromagnetic calorimeter, 9 - yoke, not shown muon range system

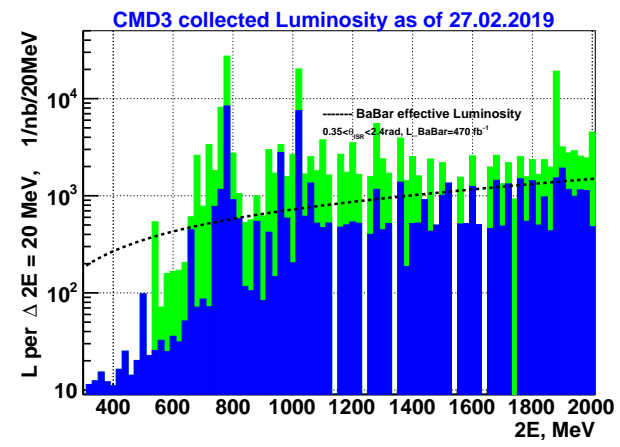

Figure 2. Luminosity collected by CMD-3. The effective ISR luminosity of $\mathrm{BaBar}$ (the last competitor experiment) is shown for comparison

with a hexagonal cell for uniform reconstruction of tracks, which is placed inside of the superconducting solenoid providing $1.3 \mathrm{~T}$ magnetic field.

The calorimetry is performed with the endcap BGO calorimeter and the barrel calorimeter. The barrel calorimeter placed outside of the superconducting solenoid consists of two systems: the inner ionisation Liquid Xenon calorimeter and the the CsI scintillation calorimeter. The total thickness of the barrel calorimeter is about $13.5 X_{0}$. The LXe calorimeter has seven layers with strip readout which give information about a shower profile and are also able to measure coordinates of photons with about millimeter precision.

During 2013-2016 the VEPP-2000 collider and the detectors have been upgraded. Starting from 2017 the accelerator complex is running with a new injection facility, which helps to solve a problem of deficit of positrons. Also the "Beamshaking" technique was introduced in 2018, which suppresses beam instabilities by introducing controllable additional kicking of a beam. As a result of such improvements, a luminosity was increased by $\sim 5$ times at middle and high energies, but is still lower than the design value by a factor of two at top energies. The $4 \times 10^{31} \mathrm{~cm}^{-2} \mathrm{~s}^{-1}$ luminosity was reached by the VEPP-2000 collider. The already collected integrated luminosity is $200 \mathrm{pb}^{-1}$ per detector, with about $135 \mathrm{pb}^{-1}$ above the $\phi$ energy and $65 \mathrm{pb}^{-1}$ from a scan below the $\phi$ (Fig. 2).

All major channels are under analysis with up to 7 pions or 2 kaons and 3 pions in the final state. The CMD-3 collaboration has already published many results such as: $e^{+} e^{-} \rightarrow p \bar{p}$, $2\left(\pi^{+} \pi^{-}\right), 3\left(\pi^{+} \pi^{-}\right), 3\left(\pi^{+} \pi^{-}\right) \pi^{0}, \omega \eta, \eta \pi^{+} \pi^{-} \pi^{0}, \omega \pi^{+} \pi^{-} \pi^{0}, K^{+} K^{-}, K_{S} K_{L}, K^{+} K^{-} \pi^{+} \pi^{-}$, search of $e^{+} e^{-} \rightarrow \eta^{\prime}$. Some more almost completed results will be soon ready for publication: $K^{+} K^{-} \eta$, $K^{+} K^{-} \omega, \omega \pi^{+} \pi^{-}, \eta \pi^{+} \pi^{-}$, search of $e^{+} e^{-} \rightarrow D_{0}^{*}$. Many others analyses are in progress, some of them will be discussed below. Status of some of them is presented in dedicated papers for proceedings of the PhiPsi19 conference:

- "An amplitude analysis of the process $e^{+} e^{-} \rightarrow 4 \pi$ ",

- "Identification of the $e^{+} e^{-} \rightarrow n \bar{n}$ events",

- "The NNbar and multihadron production at the threshold",

- "Current status of luminosity measurement with the CMD-3",

- "Study of $\pi^{+} \pi^{-} \pi^{+} \pi^{-}$production", 
- "Search for the process $e^{+} e^{-} \rightarrow D_{0}^{*}(2007)$ ",

- "Study of the process $e^{+} e^{-} \rightarrow \pi^{+} \pi^{-} \gamma$ ",

- "Study of the process $e^{+} e^{-} \rightarrow K^{+} K^{-} \pi^{0}$ ",

- "Study of the $e^{+} e^{-} \rightarrow K_{S} K_{L} \pi^{0}$ process".

\section{$2 e^{+} e^{-} \rightarrow \pi^{+} \pi^{-}$channel}

The dominant contribution to production of hadrons in the energy range $\sqrt{s}<1 \mathrm{GeV}$ comes from the $e^{+} e^{-} \rightarrow \pi^{+} \pi^{-}$mode. This channel gives the main contribution to the hadronic term and overall theoretical precision of the anomalous magnetic moment of the muon $g-2$. It is the most challenging channel because of a high-precision requirement, necessitating a systematic precision of $0.2 \%$ to fulfill requirements of new g-2 experiments and physics at future electron-positron colliders. The CMD-3 has plans to further reduce a systematic uncertainty achieved by CMD-2. Two energy scan below $1 \mathrm{GeV}$ for the $\pi^{+} \pi^{-}$measurement was performed at VEPP-2000 in 2013 and 2018. The collected data sample corresponds to about $63 \mathrm{pb}^{-1}$ of integrated luminosity with $18 \mathrm{pb}^{-1}$ during the first scan and $45 \mathrm{pb}^{-1}$ during the second one. It is already higher than in any other experiments like previous CMD-2, the BaBar[6] and the KLOE[7, 8] experiments (Fig. 3).

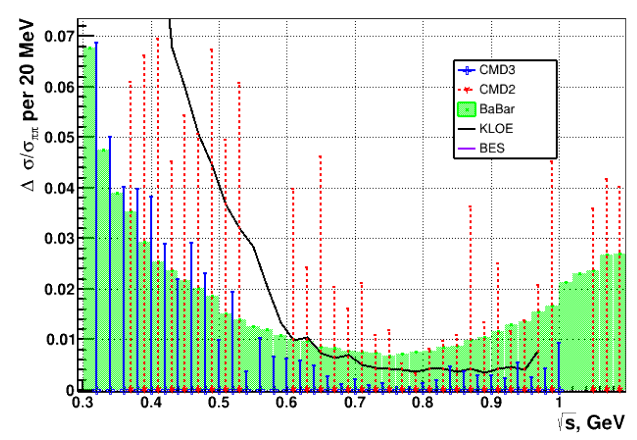

Figure 3. Statistical precision of $\left|F_{\pi}\right|^{2}$ from the CMD-3 data collected during the 2013 and 2018 seasons in comparison with the results from CMD-2, BaBar, KLOE and BESIII

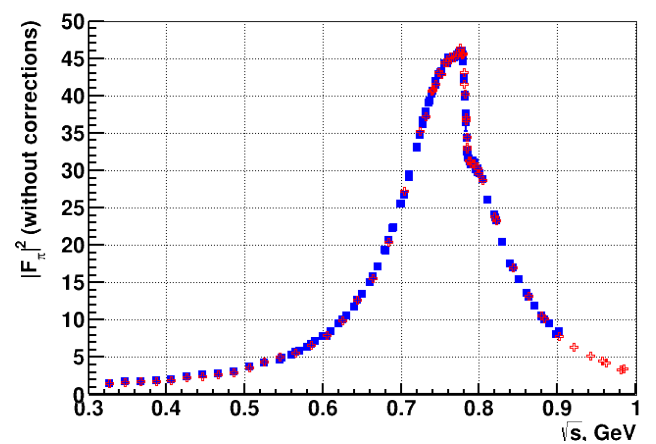

Figure 4. Preliminary results on $F_{\pi}^{2}$ from CMD3. Open crosses - separation done using the calorimeter information, filled squares - using particle momentum. Some additional corrections, common to two methods, are not applied

The crucial pieces of analysis to reach the claimed goal include stable $e / \mu / \pi$ separation, precise fiducial volume determination, theoretical precision of radiative corrections, etc. An important point is that many systematic studies rely on high collected statistics.

The $\pi^{+} \pi^{-}$process has a simple event signature with two back-to-back charged particles. They can be selected by using the following criteria: two collinear well reconstructed charged tracks are detected, these tracks are close to the interaction point, both tracks are inside a good region of the drift chamber. The selected data sample includes events with $e^{+} e^{-}, \mu^{+} \mu^{-}, \pi^{+} \pi^{-}$ pairs and cosmic muons, and it practically doesn't contain any other physical background at energies $\sqrt{s}<1 \mathrm{GeV}$. These final states can be separated using either the information about energy deposition in the calorimeter or that about particle momenta in the drift chamber. At low energies the momentum resolution of the drift chamber is sufficient to separate different types of particles. The pion momentum is well aside from the electron one up to energies 
$\sqrt{s} \lesssim 0.9 \mathrm{GeV}$, while the $\mu^{+} \mu^{-}$events are separated from others up to $\sqrt{s} \lesssim 0.66 \mathrm{GeV}$. At higher energies the peak of an electron shower in the calorimeter is far away from the peak of minimal ionization particles. Separation using energy deposition works better at higher energies and becomes less robust at lower energies.

A full energy deposition in combined LXe and CsI calorimeters is used at the moment. Further methods are under development to exploit full power of the layered barrel calorimeter. Additional information from independent measurements of energy deposition in seven strip layers of the LXe and in the CsI calorimeter gives better discrimination power between different particles due to different interaction process involved (electromagnetic shower, ionization process, nuclear interaction).

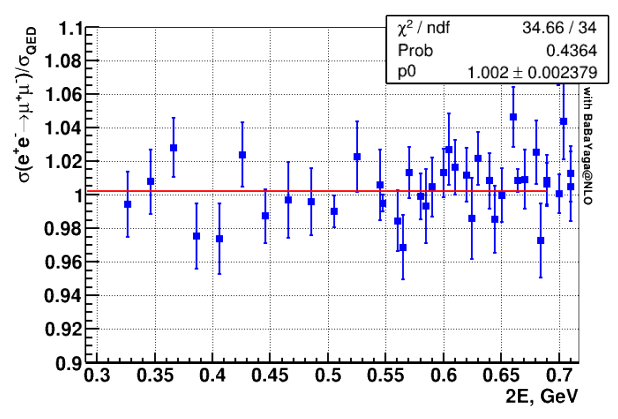

Figure 5. Preliminary results of the measurement of muon pair production in comparison with the QED prediction

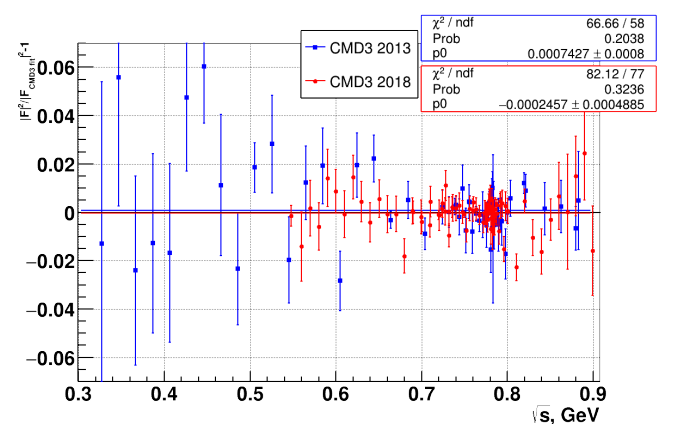

Figure 6. Ratio of the measured $2 \pi$ cross-section to the common fit $\left|F_{\pi}\right|^{2}$ parametrization. Blue points - season 2013, red points - 2018.

The preliminary result on the pion formfactor measured by the CMD-3 is shown in Fig. 4 comparing two approaches using either momentum information or energy deposition. The additional corrections, common to two methods (e.g., the trigger efficiency), are not applied. These two methods overlap in the wide energy range and provide a cross-check of each other, allowing to reach in future a systematic error of event separation at the level of $0.2 \%$.

The contribution to systematic precision from $e / \mu / \pi$ separation at the peak of the $\rho$ resonance is estimated now as $0.2 \%$ when using momentum information and up to $1 . \%$ in case of energy deposition, where in the second case work is still in progress and this number represents a current level of studies. Comparison of both methods is an important step before publishing first results.

The current estimated systematic uncertainty is about $0.65 \%$ at the $\rho$-peak and up to $0.9 \%$ at lowest points.

The comparison of results from two seasons of 2013 and 2018 is shown in Fig. 6 (from the analysis based on momentum information). Good agreement is seen at the level of $0.1 \%$, while the condition of the DCH was very different, like a different level of correlated noise, one HV layer was off in 2013 and so on, which affect very stronlgy applied corrections.

One of the tests in this analysis is a measurement of the $e^{+} e^{-} \rightarrow \mu^{+} \mu^{-}$cross section at low energy, where separation was performed using momentum information. Preliminary results of this test are consistent with the QED prediction with an overall precision of $0.25 \%$ as shown in Fig. 5.

For some sources of systematics there is a clear way of how to bring it down. $e / \mu / \pi$ separation should be greatly improved with exploiting full power of the combined barrel calorimeter. The $0.3 \%$ systematic contribution coming from the pion specific losses like 
nuclear interactions and decays in flight will be improved with better understanding of the drift chamber (which includes a detailed description of per cell inefficiencies, noise level, etc) and possible dedicated study based on the $3 \pi$-channel.

Another important source of systematics is a theoretical precision of radiative corrections [9], which contribution is estimated as $0.45 \%$ in case of event separation based on momentum information and is mainly coming from the theoretical prediction of momentum spectra from differential cross sections. Additional studies like crosschecks of different calculation approaches and further proof from comparison with experimental data are necessary in this field. As seen from effects of two-photon contributions to momentum spectra, it becomes very desirable to have an exact NNLO $e^{+} e^{-} \rightarrow e^{+} e^{-}(\gamma \gamma)$ generator to reach precision $\lesssim 0.1 \%$. Hopefully, growing up activity in such calculations for future high-precision experiments like MuOnE [10] and FCC-ee [11] will also help our experiment.

\section{$3 e^{+} e^{-} \rightarrow K^{+} K^{-}, K_{S} K_{L}$ channels}

The largest contribution to $R(s)$ at the $\phi$ meson comes from two kaon production channels. The recent CMD-3 result for the $e^{+} e^{-} \rightarrow K_{S} K_{L}$ cross section [12] is shown in Fig. 7. This is the most precise measurement of this cross section with reached $1.8 \%$ systematic uncertainty. The data is very well consistent with previous experiments, and the obtained parameters of the $\phi$ meson are in good agreement with the PDG data.

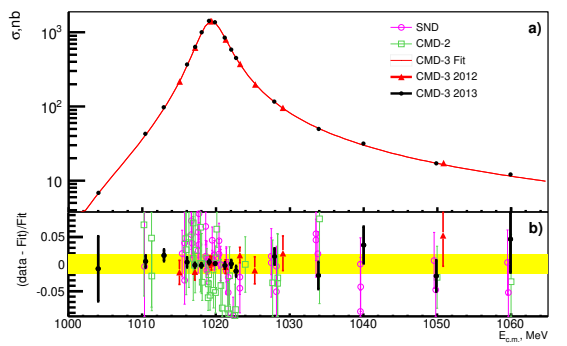

Figure 7. (a) $e^{+} e^{-} \rightarrow K_{S}^{0} K_{L}^{0}$ cross section measured in the CMD-3 experiment. (b) Relative difference between the data and fit.

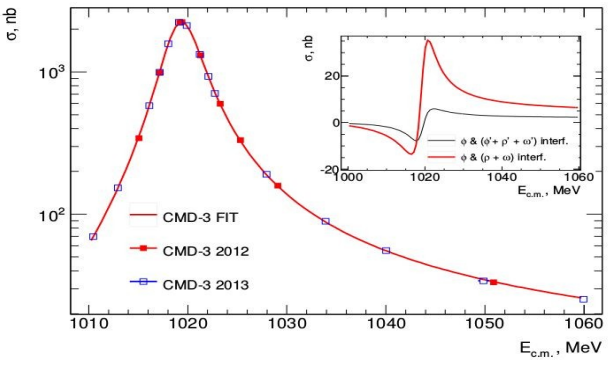

Figure 8. $e^{+} e^{-} \rightarrow K^{+} K^{-}$cross section measured by CMD-3 [14]

Results on the $e^{+} e^{-} \rightarrow K^{+} K^{-}$channel were recently published by the CMD-3 [14] experiment below $1.06 \mathrm{GeV}$ as shown in Fig. 8. This cross section was measured in the $\phi$ meson energy range with $2 \%$ systematic accuracy. As shown in Figs. 9 and 10, the obtained results have comparable accuracy, but they are not consistent, in general, with the previous data. In particular, an inconsistency between the old CMD-2 [15] result and more recent BaBar data [16] was confirmed. The new CMD-3 measurements of $e^{+} e^{-} \rightarrow K^{+} K^{-}$ and $e^{+} e^{-} \rightarrow K_{S} K_{L}$ mentioned above demonstrate good agreement with isospin symmetry: the ratio of the coupling constants with the Coulomb factor taken into account is $g_{\phi K^{+} K^{-}} / g_{\phi K_{S} K_{L}} / \sqrt{Z\left(m_{\phi}^{2}\right)}=0.990 \pm 0.017$.

The lower CMD-2 $e^{+} e^{-} \rightarrow K^{+} K^{-}$cross section is explained by overestimation of the value of the trigger efficiency for slow kaons in the previous experiment and a reanalysis of CMD-2 data is expected. 


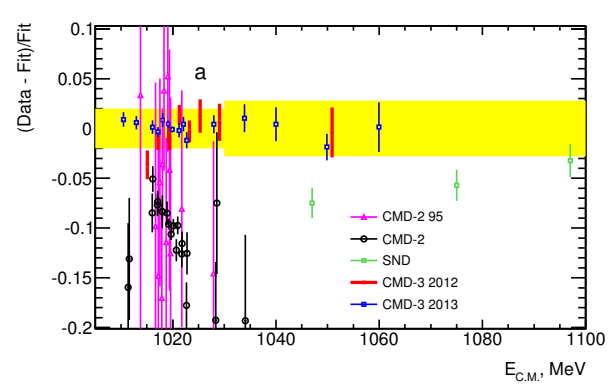

Figure 9. Comparison of the CMD-3 measurement of $e^{+} e^{-} \rightarrow K^{+} K^{-}$to previous CMD-2 [15] and SND [13] measurements. The width of the band shows the systematic uncertainties in the CMD-3 study.

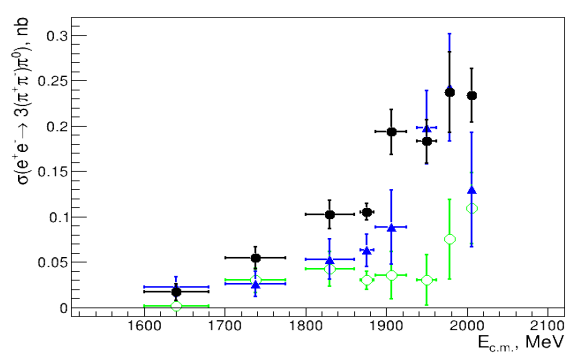

Figure 11. The $e^{+} e^{-} \rightarrow 3\left(\pi^{+} \pi^{-}\right) \pi^{0}$ cross section measured with the CMD-3 detector at VEPP2000 (dots). The contributions from the $e^{+} e^{-} \rightarrow$ $2\left(\pi^{+} \pi^{-}\right) \eta$ and $e^{+} e^{-} \rightarrow 2\left(\pi^{+} \pi^{-}\right) \omega$ reactions are shown by triangles and open circles, respectively.

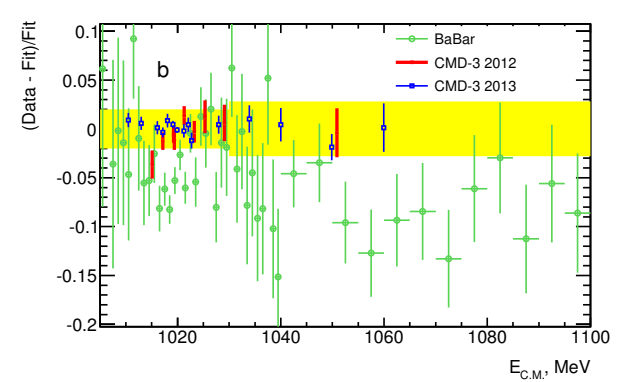

Figure 10. Comparison of the CMD-3 measurement of $e^{+} e^{-} \rightarrow K^{+} K^{-}$to the BaBar measurement [16] using the ISR approach. The width of the band shows the systematic uncertainties in the CMD-3 study.

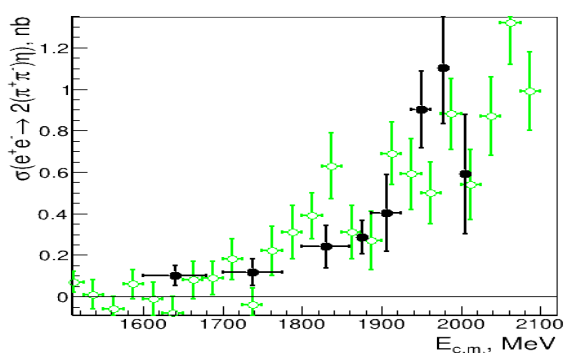

Figure 12. The $e^{+} e^{-} \rightarrow 2\left(\pi^{+} \pi^{-}\right) \eta$ cross section measured with the CMD-3 detector at VEPP2000 (circles). The results of the BaBar measurement are shown by open circles.

\section{Unaccounted modes}

The total $e^{+} e^{-} \rightarrow$ hadron cross section below $2 \mathrm{GeV}$ is calculated as a sum of exclusive cross sections for all possible hadronic modes. Some unmeasured channels are calculated using rough isospin relations, which can be very approximate. To fulfill a precise $R(s)$ measurement program, it is very important to take into account all possible channels and to do measurements of unaccounted modes or accounted by indirect isospin relations. Some of them were recently studied by the CMD-3 group.

The process $e^{+} e^{-} \rightarrow \pi^{+} \pi^{-} \pi^{0} \eta$ was studied using the $\pi^{+} \pi^{-} 4 \gamma$ final state [17]. This channel can go through several intermediate states. At least four mechanisms of this reaction: $\omega \eta, \phi \eta$, $a_{0}(980) \rho$ and structureless $\pi^{+} \pi^{-} \pi^{0} \eta$ were observed. About $50 \%$ of the total cross section in the region below $1.8 \mathrm{GeV}$ is due to the $\omega \eta, \phi \eta$ contributions. Above $1.8 \mathrm{GeV}$ the dominant mechanism of the reaction is $a_{0}(980) \rho$. The only known before $\omega \eta$ and $\phi \eta$ contributions were taken into account in the total hadron cross section, while not accounted parts give about $3-5 \%$ of the total $R(s)$ in this energy range. 
Another first time measurement of $e^{+} e^{-} \rightarrow 3\left(\pi^{+} \pi^{-}\right) \pi^{0}$ has been published recently [18] by the CMD3. The measured cross section is shown in Fig. 11, where only the $e^{+} e^{-} \rightarrow 2\left(\pi^{+} \pi^{-}\right) \eta$ subchannel was measured before by BaBar [19] as shown in Fig. 12.

\section{Conclusions}

The precise low-energy $e^{+} e^{-} \rightarrow$ hadrons cross sections are used in the SM prediction of various fundamental quantities. New precise data are desirable to fulfill requirements of new g-2 experiments and physics at future electron-positron colliders. The VEPP-2000 is now the only working collider capable of scanning energies below $<2 \mathrm{GeV}$ for a measurement of exclusive $e^{+} e^{-} \rightarrow$ hadrons channels.

The two scans below $1 \mathrm{GeV}$ for the $\pi^{+} \pi^{-}$measurement were performed in 2013 and 2018. The already collected data sample for the cross section measurement has a few times better statistical precision than was achieved by other experiments. High statistics will allow to study and to control better different systematic contributions estimated now as $0.9 \div 0.6 \%$, and the improvement here is also expected.

Several previously unmeasured processes like $e^{+} e^{-} \rightarrow \pi^{+} \pi^{-} \pi^{0} \eta$ and $3\left(\pi^{+} \pi^{-}\right) \pi^{0}$ contributing to the total hadronic cross section below $2 \mathrm{GeV}$ have been studied.

Many analyses are ongoing at CMD-3. The overall goal of the VEPP-2000 experiments is to collect $O(1) 1 / f b$ during next 5 years, which should provide new precise results on the hadron production.

This work is supported in part by Russian Foundation of Basic Research (17-02-00847, 17-02-00897, 18-32-01020).

\section{References}

[1] P. Y. Shatunov et al., Phys. Part. Nucl. Lett. 13 (2016) no.7, 995;

D. Shwartz et al., PoS ICHEP 2016 (2016) 054; this proceedings

[2] V. M. Aulchenko et al., BUDKER-INP-2001-45

[3] B. Khazin,Nucl. Phys. Proc. Suppl. 181-182 (2008): 376

[4] M. N. Achasov et al., Nucl. Instrum. Meth. A 598 (2009) 31.

[5] S. Eidelman,Nucl.Phys.Proc.Suppl. 162 (2006): 323

[6] J. P. Lees et al., Phys. Rev. D 86 (2012): 032013

[7] F. Ambrosino et al., Phys. Lett. B 670 (2009): 285

[8] F. Ambrosino et al., Phys. Lett. B 700 (2011): 102

[9] S. Actis et al., Eur. Phys. J. C 66 (2010): 585

[10] S. Di Vita, S. Laporta, P. Mastrolia, A. Primo and U. Schubert, JHEP 1809 (2018) 016

[11] FCC CDR , M. Mangano et al. CERN-ACC-2018-0056, Geneva, December 2018.

[12] E. A. Kozyrev et al.,Phys. Lett. B 760 (2016) 314

[13] M. N. Achasov et al., Phys. Rev. D 94 (2016) no.11, 112006

[14] E. A. Kozyrev et al., arXiv:1710.02989 [hep-ex].

[15] R. R. Akhmetshin et al.,Phys. Lett. B 669 (2008) 217

[16] J. P. Lees et al.,Phys. Rev. D 88 (2013) no.3, 032013

[17] R. R. Akhmetshin et al.,Phys. Lett. B 773 (2017) 150

[18] R. R. Akhmetshin et al. [CMD-3 Collaboration], Phys. Lett. B 792 (2019) 419

[19] B. Aubert et al. [BaBar Collaboration], Phys. Rev. D 76 (2007) 092005, Erratum: [Phys. Rev. D 77 (2008) 119902] 